\title{
A Born-type approximation method for bioluminescence tomography
}

\author{
Wenxiang Cong ${ }^{\text {a) }}$ and Kumar Durairaj \\ Bioluminescence Tomography Laboratory, Department of Radiology, University of Iowa, Iowa City, \\ Iowa 52242 \\ Lihong V. Wang \\ Optical Imaging Laboratory, Department of Biomedical Engineering, Texas A\&M University, Texas, \\ Texas 77843 \\ Ge Wang ${ }^{\text {b) }}$ \\ Bioluminescence Tomography Laboratory, Department of Radiology, University of Iowa, Iowa City, \\ Iowa 52242
}

(Received 21 October 2004; revised 25 December 2005; accepted for publication 28 December 2005; published 21 February 2006)

In this paper, we present a Born-type approximation method for bioluminescence tomography (BLT), which is to reconstruct an internal bioluminescent source from the measured bioluminescent signal on the external surface of a small animal. Based on the diffusion approximation for the photon propagation in biological tissue, this BLT method utilizes the Green function to establish a linear relationship between the measured bioluminescent signal and the internal bioluminescent source distribution. The Green function can be modified to describe a heterogeneous medium with an arbitrary boundary using the Born approximation. The BLT reconstruction is formulated in a linear least-squares optimization framework with simple bounds constraint. The performance of this method is evaluated in numerical simulation and phantom experiments. (C) 2006 American Association of Physicists in Medicine. [DOI: 10.1118/1.2168293]

Key words: bioluminescence tomography (BLT), diffusion approximation, Green function, Born approximation

\section{INTRODUCTION}

The use of light emitting probes as reporters of gene expressions is a powerful noninvasive technique to reveal molecular and cellular activities in a small animal in vivo. Using such probes, a small animal model can be studied for many biomedical purposes. ${ }^{1-3}$ In bioluminescent imaging, biological entities (e.g., tumor cells, genes) are tagged with luciferase enzymes, and implanted in a small animal. When the luciferase molecules are combined with a substrate luciferin in the presence of ATP and oxygen, the photons are emitted at about $600 \mathrm{~nm}^{4}$ The produced light intensity directly depends on the concentration of the luciferin and the density of luciferase molecules. The transmission of the bioluminescent photons through the biological tissue is subject to both scattering and absorption. Due to the semitransparent nature of the biological tissue, the light penetration depth is several centimeters in that spectral range, and a significant amount of the photons can be recorded using a sensitive charge coupled device (CCD) camera. ${ }^{4,5}$ Because the biological tissue does not produce bioluminescence and autoluminescence, the background noise may be kept very low. This results in an excellent signal-to-noise ratio of the imaging process. ${ }^{5,6}$ Over past years, we have been developing bioluminescence tomography (BLT) to reconstruct the bioluminescence source distribution within a small animal. ${ }^{7}$ Specifically, bioluminescent data measured on the body surface of a small animal can be processed in reference to a corresponding micro-CT volume of the same small animal to achieve three-dimensional (3D) localization and quantification of the bioluminescence activities in the animal.

The bioluminescent photon propagation in the tissue can be well described by either the radiative transfer equation or the Monte Carlo model. ${ }^{8}$ However, neither the radiative transfer equation nor the Monte Carlo model is computationally affordable in most practical applications. Given the dominance of scattering over absorption in the light propagation inside a small animal, the diffusion approximation gives a relatively accurate light propagation model to predict the photon density. ${ }^{9,10}$ Recently, based on the diffusion equation, finite element-based methods have been presented to solve the BLT problem. ${ }^{11-13}$ In the finite element scheme, the diffusion equation is discretized to yield the corresponding matrix equation, and this inverse source problem is formulated in the linear least squares framework with simple bounds constraint. The precision of the finite element solution to the diffusion equation depends on the finite element model, the element size, and the interpolation shape function. In the next section, based on the diffusion equation we develop a BLT algorithm using the analytic Green function and Born approximation. In the third section, we report numerical and experimental results. Finally, we discuss a few relevant issues, and conclude the paper.

\section{RECONSTRUCTION METHOD}

\section{A. Diffusion model}

In bioluminescent imaging, bioluminescence sources are biotechnically induced inside a small animal. In the highly 
scattering biological tissue, the diffusion approximation to gives a quite accurate description of the bioluminescent light propagation in the small animal, ${ }^{9,10,14}$

$$
\begin{aligned}
& -\nabla \cdot[D(\mathbf{r}) \nabla \Phi(\mathbf{r})]+\mu_{a}(\mathbf{r}) \Phi(\mathbf{r})=S(\mathbf{r}) \quad(\mathbf{r} \in \Omega), \\
& D(\mathbf{r})=\left\{3\left[\mu_{a}(\mathbf{r})+(1-g) \mu_{s}(\mathbf{r})\right]\right\}^{-1},
\end{aligned}
$$

where $\Omega$ denotes the region of interest for the object, $\Phi(\mathbf{r})$ the photon density (Watts $\left./ \mathrm{mm}^{2}\right), S(\mathbf{r})$ the energy density distribution of a light source (Watts $\left./ \mathrm{m}^{3}\right), D(\mathbf{r})$ diffusion coefficient, $\mu_{a}(\mathbf{r})$ the absorption coefficient $\left(\mathrm{mm}^{-1}\right), \mu_{s}(\mathbf{r})$ the scattering coefficient $\left(\mathrm{mm}^{-1}\right)$, and $g$ the anisotropy parameter. The optical parameters $\mu_{a}(\mathbf{r}), \mu_{s}(\mathbf{r})$, and $g$ can be independently determined; for example, from the literature or the diffuse optical tomography technique. Assuming that the experiment is performed in an ideal dark environment, and no photon comes in an inward direction at the boundary. Taking into account the mismatch between the refractive indices $n$ with $\Omega$ and $n^{\prime}$ in the surrounding medium, the boundary condition can be expressed as ${ }^{14,15}$

$$
\Phi(\mathbf{r})+2 C_{n d}(\mathbf{r}) D(\mathbf{r})[\nu \cdot \nabla \Phi(\mathbf{r})]=0 \quad(\mathbf{r} \in \partial \Omega),
$$

where $\boldsymbol{\nu}$ is the unit outer normal on $\partial \Omega, C_{n d}(\mathbf{r})=[1$ $+R(\mathbf{r})] /[1-R(\mathbf{r})]$. In the experiment, the medium surrounding $\Omega$ is air, for which $n^{\prime}$ is approximately 1 . Therefore, $R(\mathbf{r})$ only depends on the refractive index $n$ of the medium, and can be approximated by $R \approx-1.4399 n^{-2}+0.7099 n^{-1}$ $+0.6681+0.0636 n$. The measured quantity is the outgoing photon density on $\partial \Omega,^{15}$

$$
Q(\mathbf{r})=-D(\mathbf{r})[\nu \cdot \nabla \Phi(\mathbf{r})]=\frac{1}{2 C_{n d}(\mathbf{r})} \Phi(\mathbf{r}) \quad(\mathbf{r} \in \partial \Omega)
$$

\section{B. Reconstruction formula}

Clearly, BLT is to reconstruct the 3D source distribution $S(\mathbf{r})$ from the two-dimensional (2D) measured outgoing photon density $Q(\mathbf{r})$ on $\partial \Omega$ based on (1)-(3). This is typical underdetermined and ill-posed problem, and much more difficult than the associated forward problem. Wang et al. discussed the solution uniqueness for the BLT problem under some practical constraint conditions, and established that the unique solution or semiunique solution is possible by incorporating sufficiently a priori knowledge, including the optical parameters of the anatomy, the region and form of the light source. ${ }^{16}$

According to the partial differential equation theory, the solution to (1)-(3) can be expressed in terms of its Green function $G\left(\mathbf{r}_{d}, \mathbf{r}_{s}\right)$,

$$
\Phi\left(\mathbf{r}_{d}\right)=\int_{\Omega_{s}} S\left(\mathbf{r}_{s}\right) G\left(\mathbf{r}_{d}, \mathbf{r}_{s}\right) \mathrm{d} \mathbf{r}_{s} \quad\left(\mathbf{r}_{s} \in \Omega_{s}, \mathbf{r}_{d} \in \partial \Omega\right),
$$

where $S\left(\mathbf{r}_{s}\right)$ is the light source density at location $\mathbf{r}_{s}, \Omega_{s}$ is a subregion $\left(\Omega_{s} \subset \Omega\right)$ to be predetermined from a priori knowledge, in which a bioluminescent source distribution may present, and $\mathbf{r}_{d}$ is the detector position. The Green function $G\left(\mathbf{r}_{d}, \mathbf{r}_{s}\right)$ can be numerically computed; for example, using finite differences, finite elements or Monte Carlo method. While the direct computation is expensive, a Borntype approximation approach can be used to simplify the procedure for the Green function $G\left(\mathbf{r}_{d}, \mathbf{r}_{s}\right)$. In an infinite homogeneous medium, the Green function has an analytic form: ${ }^{10}$

$$
\begin{aligned}
& G_{h}\left(\left|\mathbf{r}_{d}-\mathbf{r}_{s}\right|\right)=\frac{\exp \left(-\mu_{\mathrm{eff}}\left|\mathbf{r}_{d}-\mathbf{r}_{s}\right|\right)}{4 \pi D\left|\mathbf{r}_{d}-\mathbf{r}_{s}\right|}, \\
& \nabla_{\zeta} G_{h}(|\zeta-\mathbf{r}|)=-\left(\mu_{\mathrm{eff}}+\frac{1}{|\zeta-\mathbf{r}|}\right) \frac{\zeta-\mathbf{r}}{|\zeta-\mathbf{r}|} G_{h}(|\zeta-\mathbf{r}|),
\end{aligned}
$$

where $\mu_{\mathrm{eff}}=\left(\mu_{a} / D\right)^{1 / 2}$ is the effective attenuation coefficient. In order to obtain the Green function for arbitrary boundaries, we can partition the body surface of a small animal into $N$ facets, each of which has an area $\Delta S_{b}$ and a surface normal $n_{b}$. Using the Kirchhoff approximation (KA) and extrapolated boundary condition, the complete Green function $G_{b}\left(\mathbf{r}_{d}, \mathbf{r}_{s}\right)$ for a homogeneous medium and boundary condition (2) can be expressed by the Green function $G_{h}\left(\mathbf{r}_{d}, \mathbf{r}_{s}\right),{ }^{17}$

$$
\begin{aligned}
G_{b}\left(\mathbf{r}_{d}, \mathbf{r}_{s}\right)= & G_{h}\left(\mathbf{r}_{d}, \mathbf{r}_{s}\right)-\sum_{b=1}^{N} W\left(\mathbf{r}_{d}, \mathbf{r}_{b}\right) G_{h}\left(\left|\mathbf{r}_{b}-\mathbf{r}_{d}\right|\right) \\
& \times\left[G_{h}\left(r_{1}\right)-G_{h}\left(r_{2}\right)\right] \Delta S\left(\mathbf{r}_{b}\right), \\
W\left(\mathbf{r}_{d}, \mathbf{r}_{b}\right)= & \left(\frac{1}{2 C_{n d} D}-\rho\left(\left|\mathbf{r}_{d}-\mathbf{r}_{b}\right|\right) \mathbf{n}_{b} \cdot\left(\mathbf{r}_{d}-\mathbf{r}_{b}\right)\right),
\end{aligned}
$$

where $r_{1}=\left|\mathbf{r}_{s}-\mathbf{r}_{b}\right|, r_{2}=\sqrt{4 C_{n d} D\left[C_{n d} D-\left(\mathbf{r}_{s}-\mathbf{r}_{b}\right) \cdot \mathbf{n}_{b}\right]+r_{1}^{2}}$, and function $\rho(r)$ is defined as $\rho(r)=\left[\mu_{\text {eff }}+(1 / r)\right](1 / r)$. Since a real animal body is not homogeneous, it should be considered as heterogeneous medium. According to the perturbation theory, ${ }^{18}$ the optical parameters may be decomposed into background values $\left(D^{0}\right.$ and $\left.\mu_{a}^{0}\right)$ and its perturbation value $\left(\delta D\right.$ and $\left.\delta \mu_{a}\right)$, that is $D(r)=D^{0}+\delta D(r), \mu_{a}(r)=\mu_{a}^{0} \delta \mu_{a}(r)$. Hence, the Green function in the heterogeneous medium can be expressed in an integral form ${ }^{19}$

$$
\begin{aligned}
G\left(\mathbf{r}_{d}, \mathbf{r}_{s}\right)= & G_{b}\left(\mathbf{r}_{d}, \mathbf{r}_{s}\right)+\int_{\Omega} G_{b}\left(\mathbf{r}_{d}, \zeta\right)\left[\nabla_{\zeta} \cdot\left(\delta D(\zeta) \nabla_{\zeta} G\left(\zeta, \mathbf{r}_{s}\right)\right)\right. \\
& \left.-\delta \mu_{a}(\zeta) G\left(\zeta, \mathbf{r}_{s}\right)\right] \mathrm{d} \zeta .
\end{aligned}
$$

Therefore, the desired Green function $G\left(\mathbf{r}_{d}, \mathbf{r}_{s}\right)$ can be obtained by an iterative procedure based on (7). In general, the Born method produces a relatively accurate approximation to the exact Green function $G\left(\mathbf{r}_{d}, \mathbf{r}_{s}\right)$ in the small perturbation case, and $G\left(\mathbf{r}_{d}, \mathbf{r}_{s}\right)$ in the heterogeneous medium under the boundary condition (2) can be formulated as ${ }^{20}$

$$
\begin{aligned}
G\left(\mathbf{r}_{d}, \mathbf{r}_{s}\right)= & G_{b}\left(\mathbf{r}_{d}, \mathbf{r}_{s}\right)-\int_{\Omega} G_{b}\left(\mathbf{r}_{d}, \zeta\right) G_{b}\left(\zeta, \mathbf{r}_{s}\right) \delta \mu_{a}(\zeta) \mathrm{d} \zeta \\
& -\int_{\partial \Omega} G_{b}\left(\mathbf{r}_{d}, \zeta\right) G_{b}\left(\zeta, \mathbf{r}_{s}\right) \frac{\delta D(\zeta)}{2 C_{n d} D(\zeta)} \mathrm{d} \zeta \\
& -\int_{\Omega}\left[\nabla_{\zeta} G_{b}\left(\mathbf{r}_{d}, \zeta\right)\right] \cdot\left[\nabla_{\zeta} G_{b}\left(\zeta, \mathbf{r}_{s}\right)\right] \delta D(\zeta) \mathrm{d} \zeta .
\end{aligned}
$$


The gradients $\nabla_{\zeta} G\left(\mathbf{r}_{d}, \zeta\right)$ and $\nabla_{\zeta} G\left(\zeta, \mathbf{r}_{s}\right)$ in (8) are obtained according to the following formulas:

$$
\begin{aligned}
\nabla_{\zeta} G_{b}\left(\mathbf{r}_{d}, \zeta\right)= & \nabla_{\zeta} G_{h}\left(\mathbf{r}_{d}, \zeta\right)+\sum_{b=1}^{N}\left[W\left(\mathbf{r}_{d}, \mathbf{r}_{b}\right) G_{h}\left(\left|\mathbf{r}_{b}-\mathbf{r}_{d}\right|\right)\right. \\
& \left.\times \mathbf{P}\left(r_{1}, r_{2}\right) \Delta S\left(\mathbf{r}_{b}\right)\right], \\
\mathbf{P}\left(r_{1}, r_{2}\right)= & {\left[G_{h}\left(r_{1}\right) \rho\left(r_{1}\right)-G_{h}\left(r_{2}\right) \rho\left(r_{2}\right)\right]\left(\zeta-\mathbf{r}_{p}\right) } \\
+ & 2 C_{n d} D G_{h}\left(r_{2}\right) \rho\left(r_{2}\right) \mathbf{n}_{b}
\end{aligned}
$$

and

$$
\begin{aligned}
\nabla_{\zeta} G_{b}\left(\zeta, \mathbf{r}_{s}\right)= & \nabla_{\zeta} G_{h}\left(\zeta, \mathbf{r}_{s}\right)-\sum_{b=1}^{N} H\left(\zeta, \mathbf{r}_{b}\right) G_{h}\left(\left|\mathbf{r}_{b}-\zeta\right|\right) \\
& \times\left[G_{h}\left(r_{1}\right)-G_{h}\left(r_{2}\right)\right] \Delta S\left(\mathbf{r}_{b}\right), \\
H\left(\zeta, \mathbf{r}_{b}\right)= & \frac{\mathbf{n}_{b} \cdot\left(\zeta-\mathbf{r}_{b}\right)}{\left|\zeta-\mathbf{r}_{b}\right|^{2}}\left[\mu_{\mathrm{eff}}^{2}+3 \rho\left(\left|\zeta-\mathbf{r}_{b}\right|\right)\right]\left(\zeta-\mathbf{r}_{b}\right) \\
& -\rho\left(\left|\zeta-\mathbf{r}_{b}\right|\right)\left(\frac{\left(\zeta-\mathbf{r}_{b}\right)}{2 C_{n d} D}+\mathbf{n}_{b}\right) .
\end{aligned}
$$

Now, the linear relationship between the predicted photon density on the domain boundary and the bioluminescence source strength has been established as summarized by (4)-(10). Since BLT is an ill-posed problem, an effective approach is to find a regularized solution by minimizing the following objective function: ${ }^{21}$

$$
\begin{aligned}
& \min _{0 \leqslant S\left(\mathbf{r}_{s}\right) \leqslant U\left(\mathbf{r}_{s}\right)}\left\{\left\|Q\left(\mathbf{r}_{d}\right)-Q^{\text {meas }}\left(\mathbf{r}_{d}\right)\right\|_{\mathbf{W}}^{2}+\lambda \eta(S)\right\}, \\
& Q\left(\mathbf{r}_{d}\right)=\frac{1}{2 C_{n d}} \int_{\Omega_{s}} G\left(\mathbf{r}_{d}, \mathbf{r}_{s}\right) S\left(\mathbf{r}_{s}\right) \mathrm{d} \mathbf{r}_{s},
\end{aligned}
$$

where $\mathbf{W}$ is a weighting matrix and norm $\|\mathbf{V}\|_{\mathbf{W}}^{2}=\mathbf{V}^{\mathbf{T}} \mathbf{W} \mathbf{V}$, $U\left(\mathbf{r}_{s}\right)$ denotes an upper bound on $S\left(\mathbf{r}_{s}\right)$ to be physically meaningful, $\eta(S)$ a stabilizing functional, $\lambda$ the regularization parameter to balance $\eta(S)$ and $\left\|Q\left(\mathbf{r}_{d}\right)-Q^{\text {meas }}\left(\mathbf{r}_{d}\right)\right\|_{\mathbf{W}}^{2}$.

\section{EXPERIMENTAL RESULTS}

\section{A. Born approximation}

We designed a numerical heterogeneous highly scattering phantom. The phantom had radius $10 \mathrm{~mm}$ and height $13 \mathrm{~mm}$, and contained four kinds of materials to represent muscle $(M)$, lungs $(L)$, heart $(H)$, and bone $(B)$, respectively. The proper optical parameters were assigned to each of the four components, ${ }^{9}$ as summarized in Table I. Then, the phantom was discretized into 23400 wedge elements, 13174 nodes, and 1120 measurement datum nodes on the external surface of the phantom, as shown in Fig. 1(a).

To evaluate the accuracy associated with the Born approximation, we compared computed boundary data $Q(\mathbf{r})$ using direct computation and Born approximation according to (4) and (6)-(10). We embedded a number of point source configurations in the phantom to test the precision of Born
TABLE I. Optical parameters used in the numerical phantom.

\begin{tabular}{ccc}
\hline \hline Material & $\mu_{a}\left(\mathrm{~mm}^{-1}\right)$ & $\mu_{s}^{\prime}\left(\mathrm{mm}^{-1}\right)$ \\
\hline$M$ & 0.10 & 1.8 \\
$L$ & 0.09 & 2.3 \\
$H$ & 0.12 & 2.0 \\
$B$ & 0.11 & 1.9 \\
\hline \hline
\end{tabular}

approximation. We first put a point source of power 1.0 nanowatts at $p_{1}(-4.96,0.31,6.00)$ in the numerical phantom. Then, the direct finite element algorithm and the Born approximation method were employed to solve the diffusion equations (1) and (2) for boundary data $Q(\mathbf{r})$, respectively. Similarly, the point light source was also moved to other positions $p_{2}(2.14,2.95,6.00), \quad p_{3}(0.33,-3.02,6.00), \quad$ and $p_{4}(-0.73,-6.06,6.00)$, respectively, as shown in Fig. 1(b). Then the direct finite element algorithm and the Born approximation procedures were repeated for more datasets. The results indicate that the computed boundary data by the two methods were very close, with the maximum relative error being less than 5\%. Figure 2 shows the comparison between the finite element solution and the Born approximation solution corresponding to the four point source positions, respectively.

\section{B. Experiment with the numerical heterogeneous highly scattering phantom}

First, we evaluated the performance of the reconstruction method presented in Sec. II using the numerical heterogeneous highly scattering phantom in Sec. III A. Two variants of the model were studied, which contained a single source and double sources, respectively. In the single source model, the source consisted of seven pointlike sources, each had a power of 0.1 nanowatts. The center of the source located at $(2.4,-3.1,7.0)$ in the right lung region $(L)$ of the phantom, as shown in Fig. 3(a). In the double source model, the sources were embedded in the left and right lungs $(L)$ of the phantom, respectively. The first source was composed of seven pointlike sources, each had a power of 0.1 nanowatts. The center of the source located at $(-3.6,-2.8,7.0)$ in the left lung region. The other source was embedded in the right lung region, and was in the same position, strength and composition as in the single source model shown in Fig. 4(a). Based on the above two models, output photon density measures at the datum nodes on the phantom surface were generated using the Born approximation method. In optical imaging experiments, the inherent data noise can be optimally modeled as a Poisson distribution. However, when the photon rate is sufficiently high, the Gaussian distribution is often a very good approximation to the Poisson distribution. ${ }^{22,23} \mathrm{In}$ practice, it is common to model the measurement noise as a Gaussian distribution. To mimic real experiment data in our bioluminescent imaging test, the directly synthesized output photon density data were corrupted with $10 \%$ Gaussian noise to obtain $Q(\mathbf{x})$. Then, our reconstruction method was used to reconstruct the light source distributions in the two numeri- 


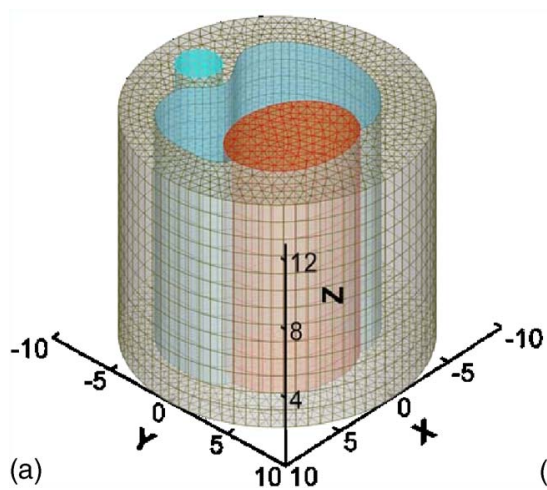

(b)

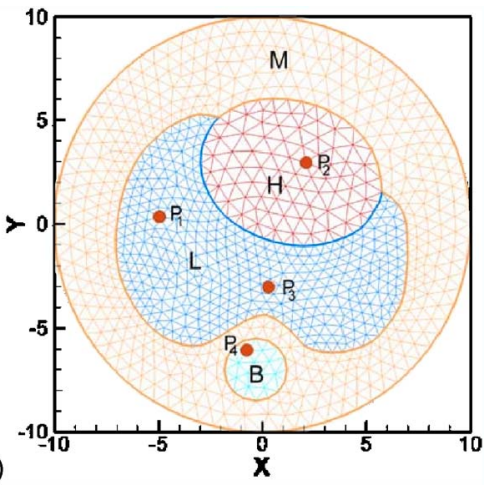

FIG. 1. Numerical heterogeneous highly scattering phantom. (a) the phantom consisting of bone $(B)$, heart $(H)$, lungs $(L)$, and tissue $(M)$; and (b) a middle cross section of the phantom. Four pointlike sources $p_{1}, p_{2}$, $p_{3}$, and $p_{4}$ locate at $(-4.96,0.31,6.00),(2.14,2.95$, $6.00),(0.33,-3.02,6.00)$, and $(-0.73,-6.06,6.00)$, respectively. The pointlike source power is always 1.0 nanowatts. cal phantoms. In the case of the single source model, the reconstructed source had a power of 0.65 nanowatts, and reconstructed source center was very close to the true one, as shown in Figs. 3(b) and 3(c). In the case of the double source model, the reconstructed source powers were 0.66 nanowatts and 0.60 nanowatts for the right and left sources, respectively. Figures 4(b) and 4(c) depicts the true and reconstructed source distributions. The reconstruction results reveal that the true sources can be recovered with about $75 \%$ of total power, while about $10 \%$ power was scattered in the neighborhood of less than $1 \mathrm{~mm}$ width surrounding the true sources. The offset of reconstructed source center was less than $1 \mathrm{~mm}$. The reconstructed source power is subject to a relative error up to $15 \%$.

\section{Experiment with the physical heterogeneous highly scattering phantom}

\section{Camera calibration}

In bioluminescent imaging, a CCD camera was used for measuring photon density data on the surface of a physical phantom or a small animal. The collected bioluminescent views must be transformed from pixel numbers into values in physical units. Hence, camera calibration is required for BLT. For that purpose, we used an absolutely calibrated integrating sphere of 8 inches in diameter, which contains a night vision monitor resolving $10 e-7 \quad \mathrm{~F}-\mathrm{L}$ ( $\sim 5$ femptowatts $/ \mathrm{mm}^{2}$ ) (LR-8-LC, 8 inches low level output sphere system, SphereOptics, Contoocook, New Hampshire). The sphere was illuminated with a tungsten lamp. A filter and variable attenuator helped select a particular wavelength with full width at half-maximum (FWHM) $20 \mathrm{~nm}$ and control the light level entering the sphere. For a selected wavelength, gray levels were associated with varying intensity values. For the wavelength range of interest 600-650 nm, a calibration formula for the CCD camera was established as $\Phi=\rho \times 0.377$ nanowatts $/ \mathrm{mm}^{2}$, where $\Phi$ represents photon density and $\rho$ the pixel value. ${ }^{13}$

\section{Physical phantom}

A heterogeneous cylindrical phantom of $30 \mathrm{~mm}$ height and $30 \mathrm{~mm}$ diameter was designed and fabricated. It consisted of four types of materials viz. high-density polyethylene (8624K16), nylon 6/6 (8538K23), delrin (8579K21), and polypropylene (8658K11) (McMaster-Carr supply com-
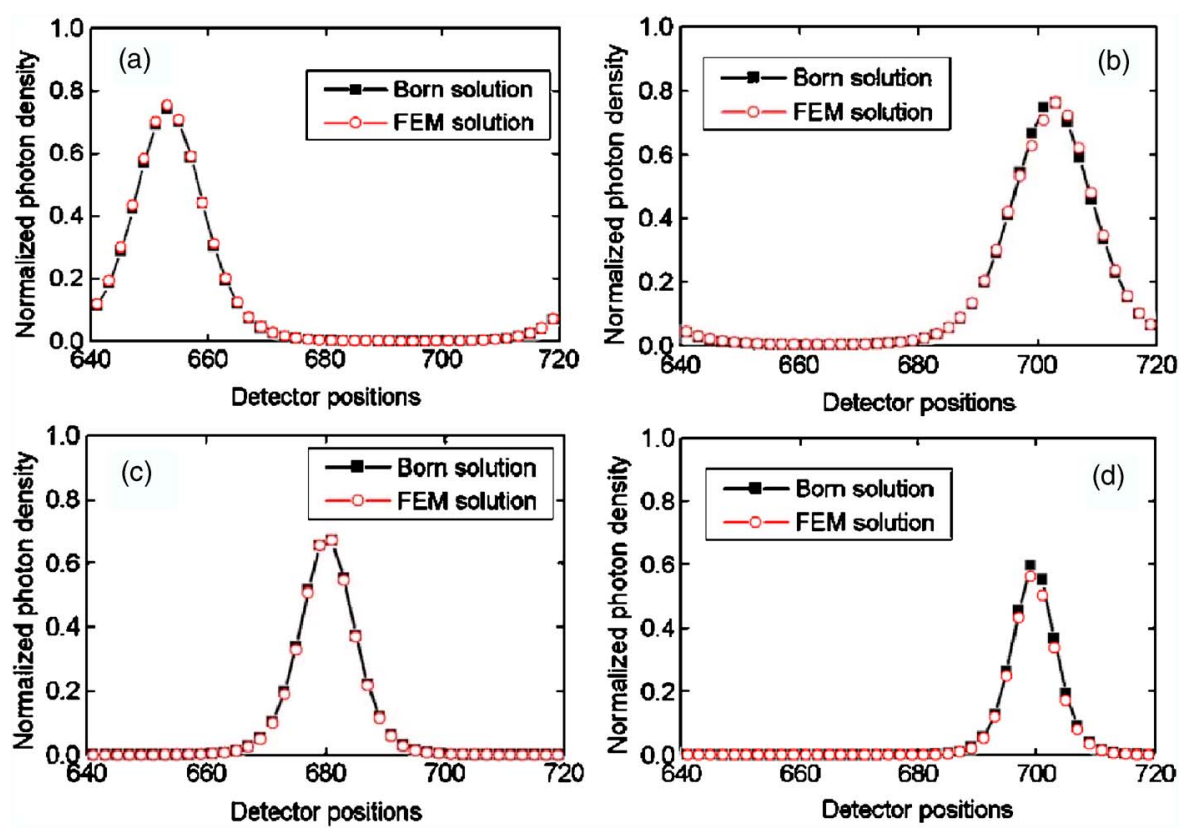

FIG. 2. Comparison between photon density profiles computed via direct computation and Born approximation along the detection circle on the phantom surface at height $8.0 \mathrm{~mm}$. (a)-(d) Results for one pointlike source at $p_{1}$, $p_{2}, p_{3}$, and $p_{4}$, respectively. 


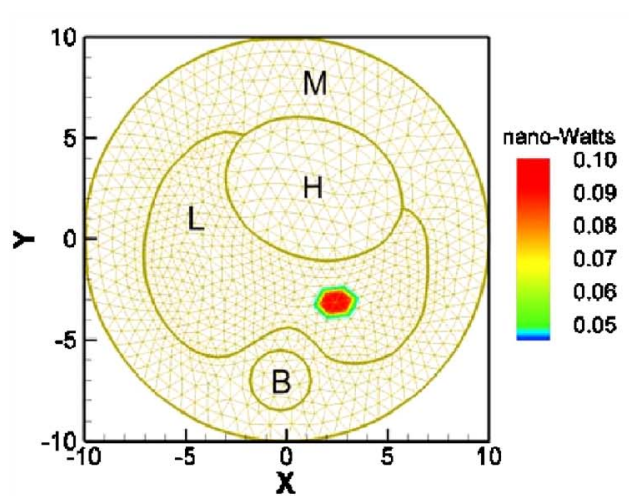

(a)

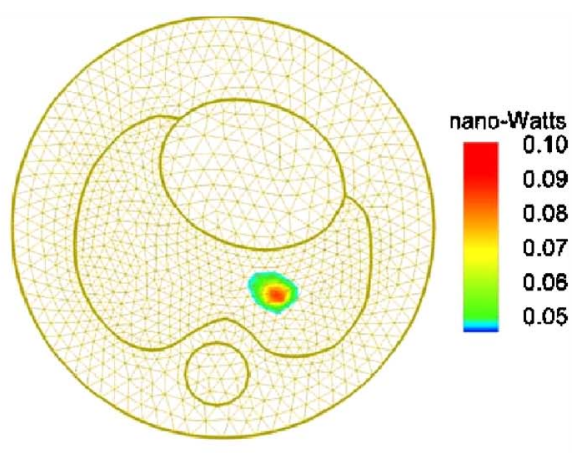

(b)

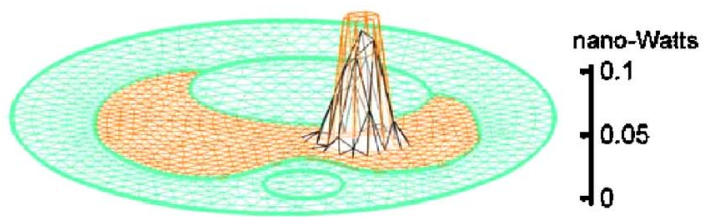

(c)

pany, Chicago, IL). The four regions $M, L, H$, and $B$ in the phantom represent the polyethylene, nylon, delrin, and polypropylene, respectively, as shown in Fig. 5(a). The luminescent light sticks (Glowproducts, Victoria, British Columbia, Canada) were used as light sources. The stick consisted of a glass vial containing one chemical solution and a larger plastic vial containing another solution with the former being embedded in the latter. By bending the plastic vial, the glass vial can be broken to mix the two solutions and emit red light around $650 \mathrm{~nm}$. Two small holes of diameter $0.6 \mathrm{~mm}$ and height $3 \mathrm{~mm}$ were drilled in the phantom with their centers at $(-9.0,1.5,15.0)$ and $(-9.0,-1.5,15.0)$ in the left $\mathrm{L}$ region of the phantom. Two catheter tubes about $1.9 \mathrm{~mm}$ height were filled with red luminescent liquid, and were placed inside the two holes as light sources, respectively.
Emitting light power of two catheter tubes was measured with the CCD camera. They are 105.1 nanowatts and 97.4 nanowatts, respectively.

\section{Optical parameters}

Since the optical parameters were needed for BLT, we had to determine them for the four components $(M, H, L$, and $B)$ of the physical phantom. Four homogeneous cylindrical phantoms with diameter $20 \mathrm{~mm}$ and height $20 \mathrm{~mm}$ were made of the above-mentioned four kinds of materials, respectively. The light about $650 \mathrm{~nm}$ was output from the exit port of the integrating sphere, and guided into a small hole with $10 \mathrm{~mm}$ depth of one specimen through the optic fiber. In a dark environment, the specimen was imaged and cap-

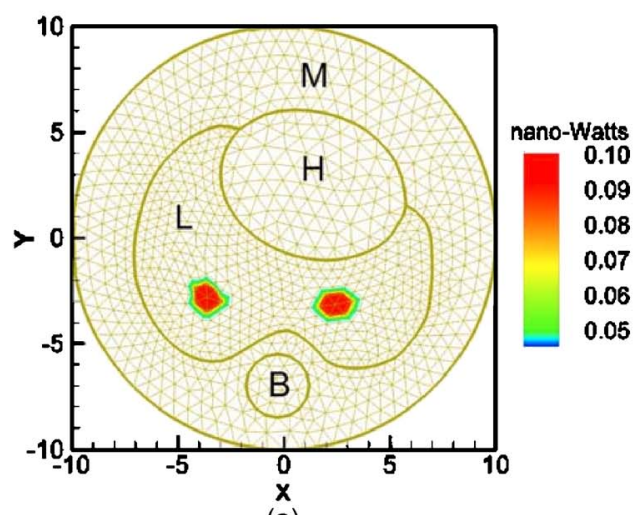

(a)

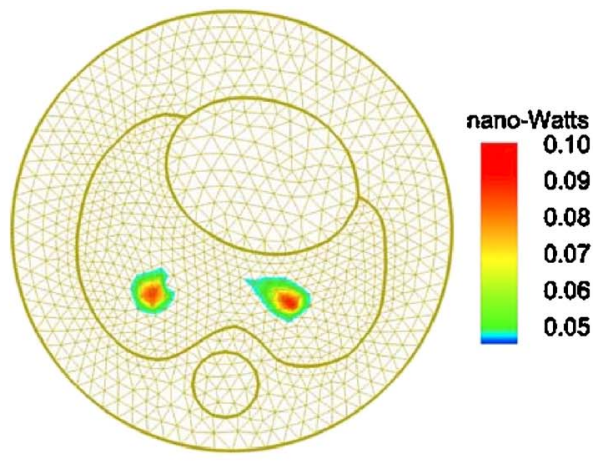

(b)

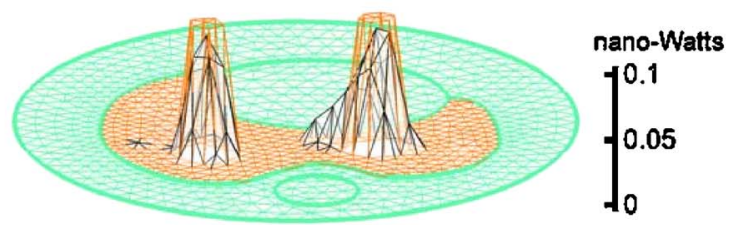

(c)

FIG. 4. BLT reconstruction of two sources in the left and right $\mathrm{L}$ region, respectively. (a) A middle crosssection of the phantom with the true source distribution, (b) 2D graphics of the reconstructed source distribution, and (c) 3D graphics of the reconstructed source distribution from the surface data corrupted by $10 \%$ Gaussian noise, and true source and reconstructed source distribution are displayed using low intensity and high intensity lines, respectively. 


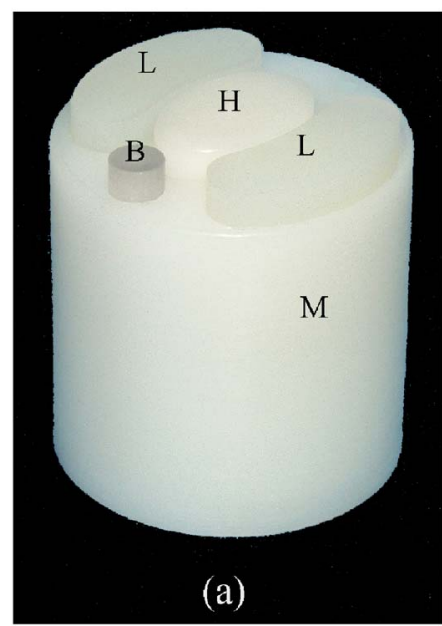

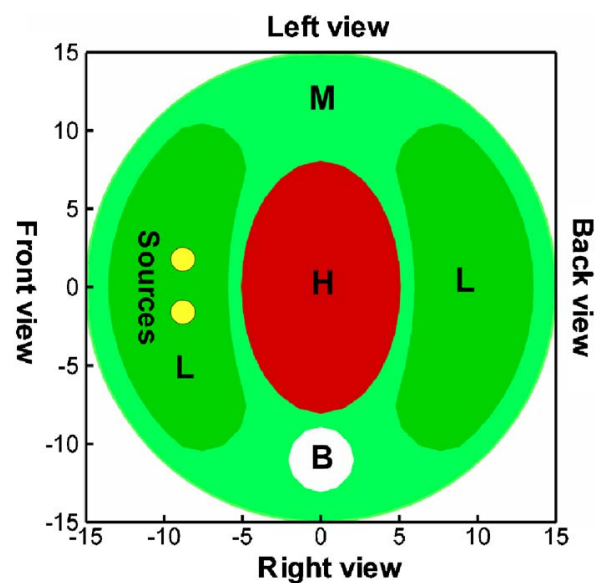

(b)
FIG. 5. Physical heterogeneous highly scattering phantom. (a) A photography of the highly scattering phantom consisting of polyethylene $(M)$, nylon $(L)$, delrin $(H)$, and polypropylene $(B)$; and (b) a middle cross section through two small hollow cylinders for hosting sources in the $\mathrm{L}$ region. tured the output photon on the other surface of the specimen using cooled CCD camera (Roper Scientific Inc, Trenton, NJ) with an exposure time of 30 seconds. Then, the surface output photon density was calculated by transforming the pixel values in the CCD image into the light unit according to our experimentally established calibration formula. The specimen was modeled as a semi-infinite homogeneous medium. The steady-state diffusion theory was applied with the extrapolated boundary condition that the photon density was zero at an artificial boundary parallel to the boundary of the medium. Then, an analytic formula was used to predict the photon density on the bottom surface. Finally, a nonlinear least square fitting was done to determine the absorption coefficient $\mu_{a}$ and the reduced scatter coefficient $\mu_{s}^{\prime}$. The calculated optical parameters of the four regions are given in Table II. ${ }^{13}$

\section{Data acquisition}

The heterogeneous phantom containing the two light sources was placed on the sample holder in front of the CCD camera. Then, the data acquisition was performed in a dark environment along four horizontal orientations 90 degrees apart. During each acquisition, one luminescent view was taken by exposing the camera for 60 seconds, as shown in Fig. 6. A permissible source region $\Omega_{s}$ was assigned by analyzing the four luminescent views taken by the CCD camera. These four planar images exhibited high value clusters near the center of the front image and a low value distribution in the back image. On the right-hand side and left-hand side images, it was seen that one side displayed high values while

TABLE II. Optical parameters estimated for the heterogeneous highly scattering phantom.

\begin{tabular}{ccc}
\hline \hline Material & $\mu_{a}\left(\mathrm{~mm}^{-1}\right)$ & $\mu_{s}^{\prime}\left(\mathrm{mm}^{-1}\right)$ \\
\hline$T$ & 0.0068 & 1.031 \\
$L$ & 0.0233 & 2.000 \\
$H$ & 0.0104 & 1.096 \\
$B$ & 0.0001 & 0.060 \\
\hline
\end{tabular}

the other side showed low values. From these observations, we infer that the light source region should be in the front part of the phantom. Along the longitudinal direction, high values were clustered around $z=15 \mathrm{~mm}$ relative to the phantom bottom. Consequently, the permissible source region was specified as

$$
\begin{aligned}
\Omega_{s} & =\{(x, y, z) \mid-12.0<x<-6.0,-7.0<y<7.0,13.0 \\
& <z<17.0\} .
\end{aligned}
$$

\section{BLT reconstruction}

To simulate the photon propagation in the phantom, a geometrical model of diameter $30 \mathrm{~mm}$ and height $20.66 \mathrm{~mm}$ was established corresponding to a middle section of the physical phantom. Based on this geometrical model, a finiteelement discrete model was built consisting of 13608 wedge elements and 7809 nodes with 1216 datum nodes on the phantom surface, as shown in Fig. 7(a). The optical properties of every element were assigned in reference to the opti-

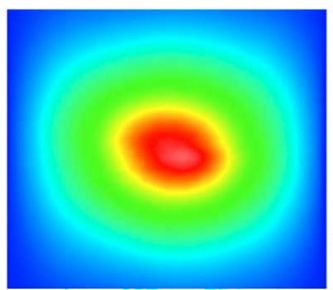

(a)

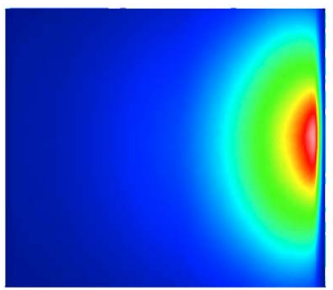

(c)

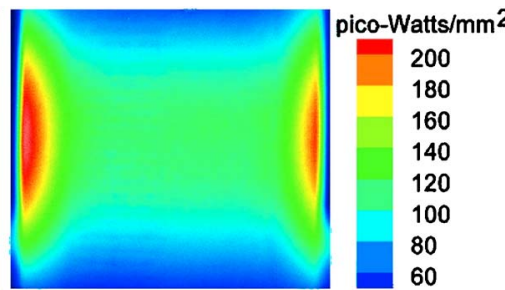

(b)

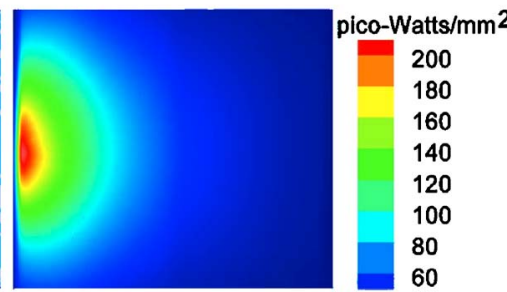

(d)
FIG. 6. Luminescent views on the cylindrical phantom surface taken using a CCD camera in four horizontal directions 90 degrees apart. (a) Front, (b) back, (c) left-hand side, and (d) right-hand side views of the phantom. 


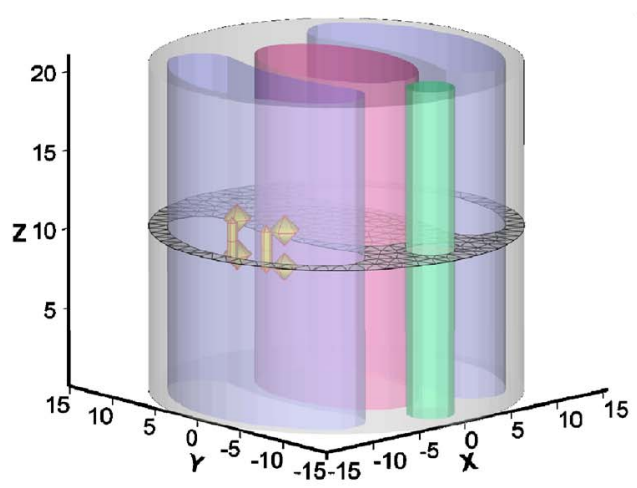

(a)

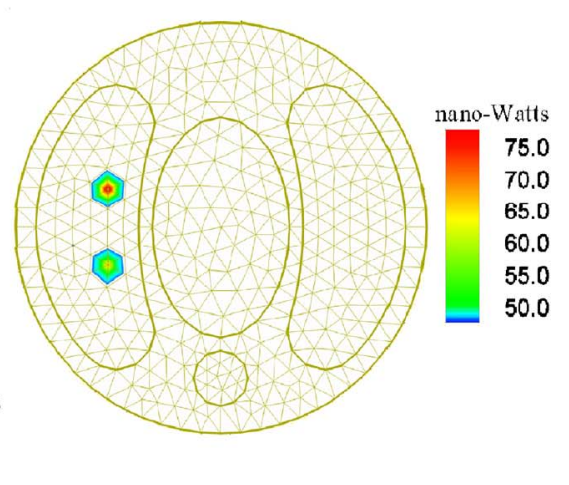

(b) cal parameters reported in Sec. III C 3. On the surface of the geometric model 19 circles were selected, separated by $1.148 \mathrm{~mm}$, along each of which 64 detection locations were uniformly distributed. The pixel values of the luminescent views were transformed into corresponding physical units. The measured photon density at each detector location was obtained from the luminescent images (Fig. 6). The computed photon density at the corresponding detection point was obtained using (3) and (4) in Sec. II. Then, the reconstruction method described in Sec. II was applied to reconstruct the light source distribution in the heterogeneous phantom. The reconstructed results correctly revealed that there were two light sources in the phantom, their center located at $(-8.24,2.75,15.0)$ and $(-8.24,-2.79,15.0)$ with total power 76.3 nanowatts and 67.6 nanowatts, respectively. Each light source just consisted of up-and-down two point-like sources separated about $2 \mathrm{~mm}$, matching the real cylinder light source inside phantom. The pointlike source at a node can be regarded as an equivalent spherical source with a corresponding power and radius estimated as half an average size of the element edges. Figure 7 shows both a photograph of the phantom and the reconstructed source distribution. The differences between the reconstructed and real source positions were less than $1.5 \mathrm{~mm}$. The relative errors in the source power were $27 \%$ and $31 \%$ for the two sources, respectively. The computed surface photon density profiles based on the reconstructed light sources were in good agreement with the experimental counterparts, with the average relative error being $11 \%$. Two representative photon density profiles for the comparison between the computed and measured photon density on the side surface of the phantom were shown in Fig. 8.

\section{DISCUSSION AND CONCLUSIONS}

The above-described results have demonstrated the feasibility of our reconstruction method for BLT. In the numerical phantom experiment, even though the measured data on the external surface of the phantom were corrupted by $10 \%$ Gaussian noise, the light sources can be located fairly accurately, and source power can be recovered up to $85 \%$. All the simulation experiments we performed have shown that the algorithm is fairly robust with respect to data noise and the initial distribution in the optimization procedure. Our physical phantom experiment has confirmed that the method can reliably identify light sources in the heterogeneous background. The error of the reconstructed source location is about $1.5 \mathrm{~mm}$. The error of the reconstructed source power is about $30 \%$. Compared with the data we obtained in the numerical simulation, the reconstruction results for the physical phantom may be further improved by using the radiative transport equation instead of the diffusion equation, decreasing the element size, reducing the measurement noise, and so on. Although the total power of each reconstructed source was close to the true power, the volumes of the reconstructed sources are different from the actual values, depending on the mesh size. Generally, the smaller the mesh size we use, the higher accuracy we will achieve in reconstructing the
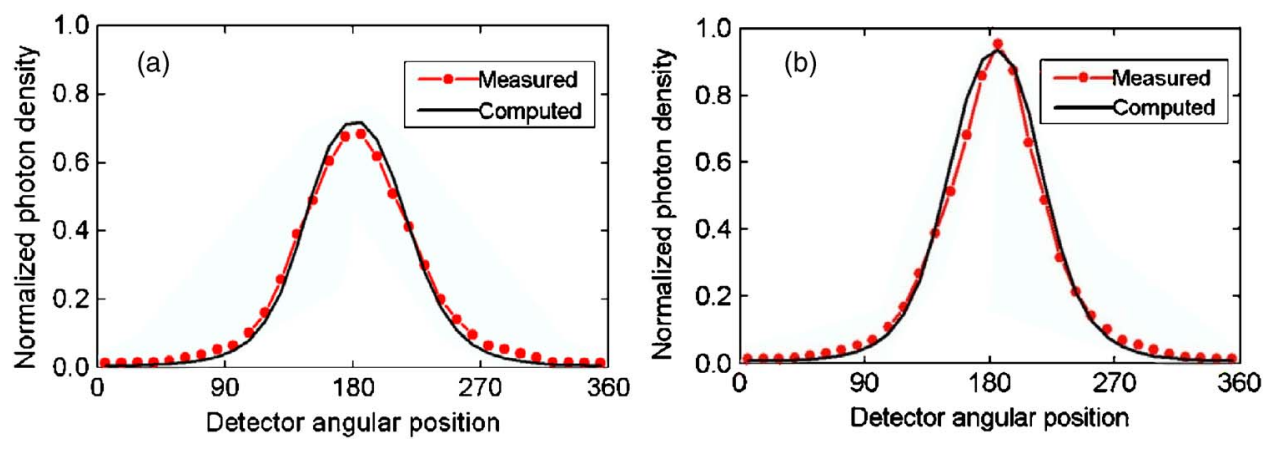

FIG. 8. Comparison between measured and computational photon density profiles along the detection circle on the phantom surface at heights (a) $10.37 \mathrm{~mm}$ and (b) $17.27 \mathrm{~mm}$. 
shape and/or size of the underlying source. A procedure for progressive mesh refinement will help enhance the reconstruction quality, and will be explored in our future study.

In this work, we have established a direct linear relation between the light source distribution and the measured photon density on the side surface of the object. The advantage over the finite element reconstruction method ${ }^{13}$ is that our method does not involve an inverse matrix, and can handle more complicated numerical models. Particularly, our method is more suitable for parallel computing. In addition, this method will be more efficient in the case of smooth varying optical properties consistent to Born approximation theory.

Currently, we are working to perform living mouse studies using the proposed reconstruction method. It would be critically important for BLT to be applied in biomedical applications. Such in vivo BLT is highly challenging, involving geometrical modeling of the mouse, determination of optical parameters, bioluminescent data acquisition, multiresolution iterative reconstruction, and generation for a finite element mesh of a heterogeneous, irregular and complicated object.

In conclusion, we have developed a Born-type approximation method for BLT, obtained encouraging preliminary results in both numerical simulation and physical phantom experiments, and established that our proposed method is effective for BLT. In vivo mouse studies using our BLT method will be reported in the future.

\section{ACKNOWLEDGMENT}

This work is supported by NIH/NIBIB Grant No. EB001685.

\footnotetext{
${ }^{a)}$ Electronic mail: cong@ct.radiology.uiowa.edu

${ }^{b}$ Electronic mail: ge-wang@ieee.org

${ }^{1}$ M. Rudin and R. Weissleder, "Molecular imaging in drug discovery and development," Nat. Rev. Drug Discovery 2, 123-131 (2003).

${ }^{2}$ S. Wang, J. Petravicz, and X. O. Breakefield, "Bioluminescence Imaging of regulated gene expression using HSV-1 Amplicon vectors in rodent brain," Molecular Therapy 9, S27 (2004).

${ }^{3}$ P. Ray, A. M. Wu, and S. S. Gambhir, "Optical bioluminescence and positron emission tomography imaging of novel fusion reporter gene in tumor xenografts of living mice," Cancer Res. 63, 1160-1165 (2003).

${ }^{4}$ C. H. Contag and M. H. Bachmann, "Advances in vivo bioluminescence
}

imaging of gene expression," Annu. Rev. Biomed. Eng. 4, 235-260 (2002).

${ }^{5}$ B. W. Rice, M. D. Cable, and M. B. Nelson, "In vivo imaging of lightemitting probes," J. Biomed. Opt. 6, 432-440 (2001).

${ }^{6}$ T. C. Doyle, S. M. Burns, and C. H. Contag, "In vivo bioluminescence imaging for integrated studies of infection," Cell. Microbiol. 6, 303-317 (2004).

${ }^{7}$ G. Wang, E. A. Hoffman, G. McLennan, L. V. Wang, M. Suter, and J. Meinel, "Development of the first bioluminescent CT scanner," Radiology 229(P), 566 (2003).

${ }^{8}$ L.-H. Wang, S. L. Jacques, and L.-Q Zheng, "MCML—Monte Carlo modeling of light transport in multi-layered tissues," Comput. Methods Programs Biomed. 47, 131-146 (1995).

${ }^{9}$ A. J. Welch and M. J. C. van Gemert, Optical and Thermal Response of Laser-Irradiated Tissue (Plenum, New York, 1995).

${ }^{10}$ S. R. Arridge, "Optical tomography in medical imaging," Inverse Probl. 15, R41-R93 (1999).

${ }^{11}$ W. Cong, D. Kumar, Y. Liu, A. Cong, and G. Wang, "A practical method to determine the light source distribution in bioluminescent imaging," Proc. SPIE 5535, 679-686 (2004).

${ }^{12} \mathrm{X}$. Gu, Q. Zhang, L. Larcom, and H. Jiang, "Three-dimensional bioluminescence tomography with model-based reconstruction," Opt. Express 12, 3996-4000 (2004).

${ }^{13}$ W. Cong, G. Wang, D. Kumar, Y. Liu, M. Jiang, L. V. Wang, E. A. Hoffman, G. McLennan, P. B. McCray, J. Zabner, and A. Cong, "Practical reconstruction method for bioluminescence tomography," Opt. Express 13, 6756-6771 (2005).

${ }^{14}$ J. J. Duderstadt and L. J. Hamilton, Nuclear Reactor Analysis (Wiley, New York, 1976).

${ }^{15}$ M. Schweiger, S. R. Arridge, M. Hiraoka, and D. T. Delpy, "The finite element method for the propagation of light in scattering media: Boundary and source conditions," Med. Phys. 22, 1779-1792 (1995).

${ }^{16} \mathrm{G}$. Wang, Y. Li, and M. Jiang, "Uniqueness theorems in bioluminescence tomography," Med. Phys. 31, 2289-2299 (2004).

${ }^{17}$ J. Ripoll, M. Nieto-Vesperinas, R. Weissleder, and V. Ntziachristos, "Fast analytical approximation for arbitrary geometries in diffuse optical tomography," Opt. Lett. 27, 527-529 (2002).

${ }^{18}$ S. Economou, Green's Functions in Quantum Physics (Springer-Verlag, New York, 1983).

${ }^{19}$ V. A. Markel, and J. C. Schotland, "Inverse problems in optical diffusion tomography. I. Fourier-Laplace inversion formulas," J. Opt. Soc. Am. A 18, 1336-1347 (2001).

${ }^{20}$ M. A. O'Leary, Dissertation, University of Pennsylvania, 1996.

${ }^{21}$ P. P. B. Eggermont, "Maximum entropy regularization for fredholm integral equation of the first kind," SIAM J. Math. Anal. 24, 1557-1576 (1993).

${ }^{22}$ J. C. Ye et al., "Optical diffusion tomography by iterative coordinateDescent optimization in a Bayesian framework," J. Opt. Soc. Am. A 16, 2400-2412 (1999).

${ }^{23}$ E. Waks, K. Inoue, W. D. Oliver, E. Diamanti, and Y. Yamamoto, "Highefficiency photon-number detection for quantum information processing," IEEE J. Sel. Top. Quantum Electron. 9, 1502-1511 (2003). 